Please do not remove this page

RMIT

UNIVERSITY

\title{
Ultra-broadband photonic antenna system with microwave photonic combination
}

Lopez-Lara, Elias; Lunn, Timothy; Fu, Wei; Ghorbani, Kamran; Rowe, Wayne; Mitchell, Arnan

https://researchrepository.rmit.edu.au/esploro/outputs/9921863686701341/filesAndLinks?institution=61 RMIT_INST\&index=null

Lopez-Lara, E., Lunn, T., Fu, W., Ghorbani, K., Rowe, W., \& Mitchell, A. (2007). Ultra-broadband photonic antenna system with microwave photonic combination. Asia Pacific Microwave Conference 2007, 1-4. https://doi.org/10.1109/APMC.2007.4554726

Published Version: https://doi.org/10.1109/APMC.2007.4554726

Repository homepage: https://researchrepository.rmit.edu.au

(c) 2007 IEEE. Personal use of this material is permitted. However, permission to reprint/republish this material for advertising or promotional purposes or for creating new collective works for resale or redistribution to servers or lists, or to reuse any copyrighted component of this work in other works must be obtained from the IEEE.

Downloaded On 2023/04/26 22:11:43 +1000 


\title{
Ultra-broadband Photonic Antenna System with Microwave Photonic Combination
}

\author{
E. R. Lopez, T. Lunn, W. Fu, K. Ghorbani, W. S. T. Rowe and A. Mitchell \\ School of Electrical and Computer Engineering \\ RMIT University \\ Melbourne, Australia \\ Email: s3008525@student.rmit.edu.au
}

\begin{abstract}
The bandwidths of two Aperture Stacked Patch antennas have been combined successfully into a single large bandwidth using microwave photonic techniques. The antenna system produces a $10 \mathrm{~dB}$ return loss bandwidth of approximately $105 \%$ with average gain level of $5 \mathrm{dBi}$ and amplitude variation of $\pm 1.5 \mathrm{~dB}$. Radiation analy sis shows the level of interaction of the two antennas according to their relative position.
\end{abstract}

Keywords - Stacked patch antenna, antenna arrays, Microwave Photonics, wavelength multiplexing

\section{INTRODUCTION}

As wireless technologies develop, different protocols operating at different frequencies have emerged as new services are being proposed. Although it is fairly simple to transfer from one protocol to another (since most modern signal processing is conducted digitally), most wireless hardware is designed to operate at a specific frequency and thus must be replaced as new wireless services are added. The replacement of this physical hardware is prohibitively expensive. It is possible to conceive a 'wireless kiosk' where hardware can operate over extremely broad bandwidths allowing services to be added as a software update $[4,5]$. The deployment of such hardware would enable very low-cost network upgrades for many decades to come.

An 'Ultra-broadband' antenna system consisting of two broadband patches combined using a diplexer has been demonstrated previously [1]. The diplexer design was nontrivial and required very delicate trimming to achieve acceptable performance. Recent investigations [2, 3] have proposed the use of photonic combination of these RF signals. Our most recent demonstration [3] exhibited appreciable interaction between the two patches at their interacting bandedges where the two antennas effectively operate as an array. This interaction must be managed to achieve acceptable gain and radiation pattern performance across the band.

This paper presents a novel ultra-broadband antenna formed from two radiating elements with Microwave Photonic (MP) signal combination, where the interacting band-edges are managed using simple RF filters. The paper is configured as follows. Section II presents the characterization of the antenna elements and an investigation of their optimal combination. Section III demonstrates the microwave photonic ultrabroadband photonic antenna system. Section IV presents an assessment of the demonstrated performance and options for future work.

\section{Two PATCH ANTENNA ELEMENTS with FILTERS}

To form the ultra-broadband antenna, two aperture stacked patch antennas $[5,6]$ have been selected. These are designed for nominal bands of 1 to $2 \mathrm{GHz}$, and 2 to $4 \mathrm{GHz}$ respectively. We predict these two antennas can be combined into a single, ultra-broadband radiating element covering from 1 to $4 \mathrm{GHz}$. The interaction between the two antennas can be significant if they are combined without managing the cross over at $2 \mathrm{GHz}$. At this intermediate frequency, both elements radiate and the pair will operate as a poorly balanced two element array with detrimental consequences for the gain and radiation pattern [3].

To reduce the interaction between the elements at $2 \mathrm{GHz}$, simple filtering has been introduced to each antenna. A low pass stepped impedance filter and a band pass double stub filter were used on the low and high frequency patches respectively. Both filters were designed using Agilent's Advance Design System (ADS) and realised on a Rogers RT/Duroid substrate. Both filter/antenna arrangements are characterized using an indoor anechoic measurement facility.

The combined filter/antennas were first measured in isolation. The gain characterization of the two antennas ( $\mathrm{RF}$ ASP1 and RF ASP2) measured in isolation is shown as part of Fig. 1. The radiation patterns for these two antennas are presented in Fig 2. Fig. 2a) presents ASP1 radiating at $1.5 \mathrm{GHz}$ and Fig. 2b) presents ASP2 radiating at $3 \mathrm{GHz}$. Characteristic stacked patch radiation performance is evident with the front to back ratio of more than $6 \mathrm{~dB}$ consistent with those in [1]

To investigate their interaction, the antennas were then mounted side by side. Each antenna was characterised with the other terminated in a $50 \Omega$ load. The gain response of each element was recorded. Fig. 1 shows the vector sum of these two measurements for phase offsets of $0,45,90$ and $180^{\circ}$. It is clear that the least disturbance to the gain response at the cross over point of $1.9 \mathrm{GHz}$ occurs with $0^{\circ}$ phase shift.

The predicted gain response of the combined antennas extends from $1.2 \mathrm{GHz}$ to around $3.8 \mathrm{GHz}$. The average gain of the in-phase predicted response is around $7 \mathrm{dBi}$ with an amplitude variation of $\pm 1 \mathrm{~dB}$. The predicted response at $180^{\circ}$ phase difference highlights the maximum interaction between the two filter/antenna arrangements at the cross-over point.

The radiation patterns of the two antennas were recorded at the cross over frequency of $1.9 \mathrm{GHz}$. These radiation patterns were then combined as a vector sum with $0^{\circ}$ phase offset. This combined radiation pattern is presented in Fig. 2c). 


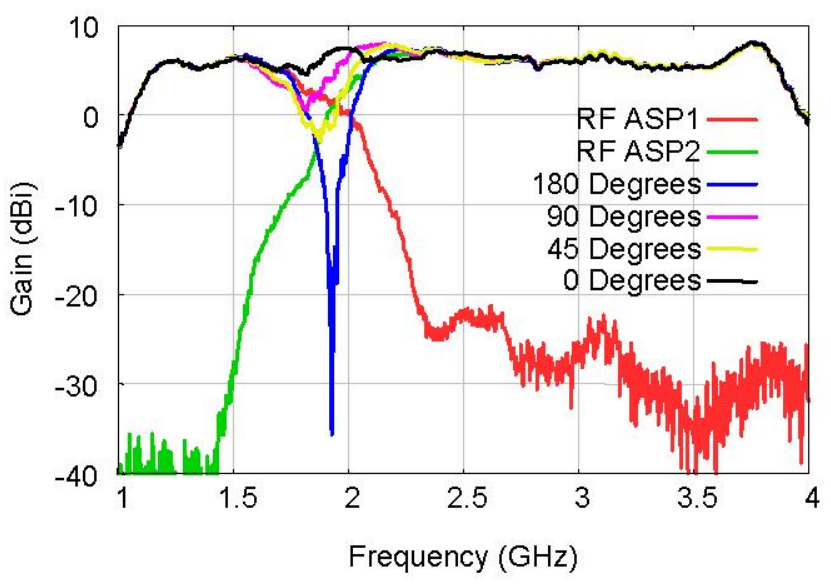

Figure 1. Measured gain of the independent filter/antennas and the predicted combined response

The difference between the main lobe power level and the side lobes is over $10 \mathrm{~dB}$. These results show a very good approximation to the ones presented in [1] using a diplexer.

The excellent gain response and acceptable radiation pattern at $1.9 \mathrm{GHz}$ are encouraging, suggesting that combining these two elements will indeed produce a single, ultrabroadband patch. Of particular importance is the fact that the simple filters provide equivalent performance to the diplexer of [1]. It now remains to demonstrate that the two elements can be summed with minimal distortion or interaction.

\section{Microwave Photonic Combination}

In this section, we propose the use of a Microwave Photonic (MP) system to combine the two antennas as depicted in Figure 3. The antenna elements (ASP1 and ASP2) were connected to their respective filters (as described in Section II) and mounted side by side in the anechoic chamber. The filter outputs are connected to two broadband optical modulators via mechanically variable phase trimmers. Each modulator is a perfect broadband termination for their respective filter/antenna, completely isolating the filters from one and other. The phase trimmers were used to adjust the vector summation of each filter/antenna output.

Two laser diodes (LD1 and LD2) deliver optical carriers at different wavelengths to the modulators using polarization maintaining fiber to ensure stability and efficiency. Since the two modulated carriers are different wavelengths, they can be combined into a single optical link without coherent interference effects using a $3 \mathrm{~dB}$ coupler $[7,8]$. This single optical carrier is amplified using an Erbium-Doped Fiber Amplifier (EDFA) before being converted by a photodetector (PD) back into the electrical domain. Finally, the PD is connected to a spectrum analyzer (SA) through a broadband $\mathrm{RF}$ amplifier. An optical spectrum analyzer monitors the optical channels and the entire configuration was computer controlled.
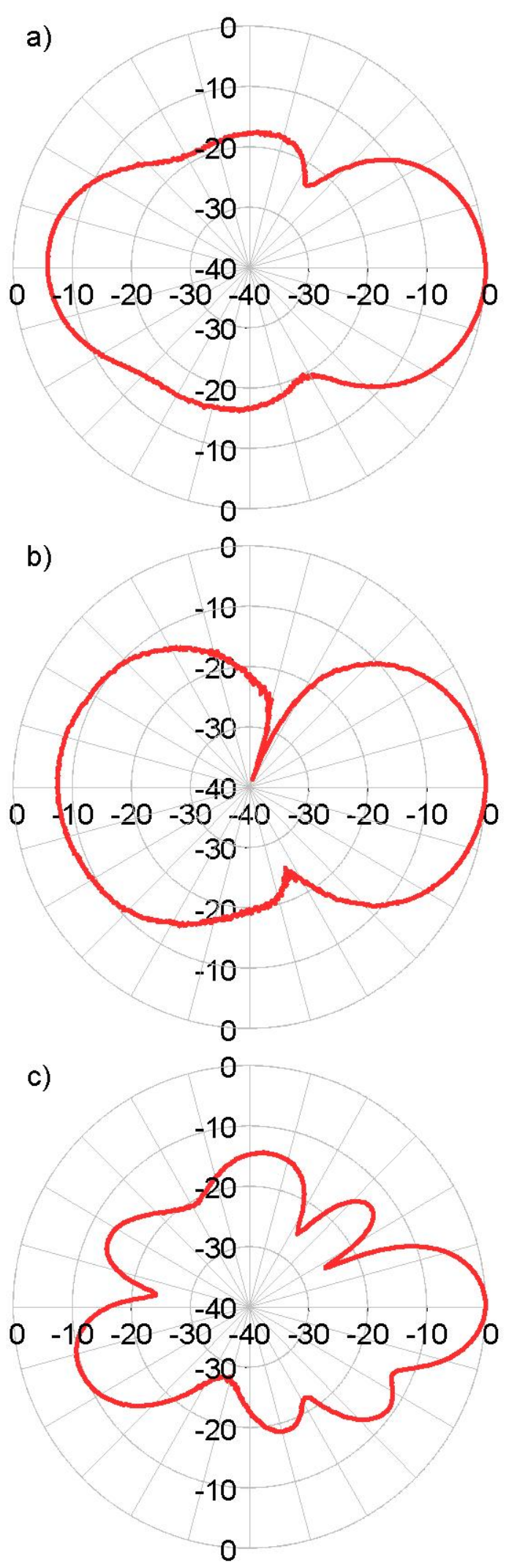

Figure 2. Measured H-plane Radiation Pattern of a) ASP1 at 1.5 $\mathrm{GHz}$ b) ASP2 at $3 \mathrm{GHz}$ c) ASP1 and ASP2 combined at $1.9 \mathrm{GHz}$ 


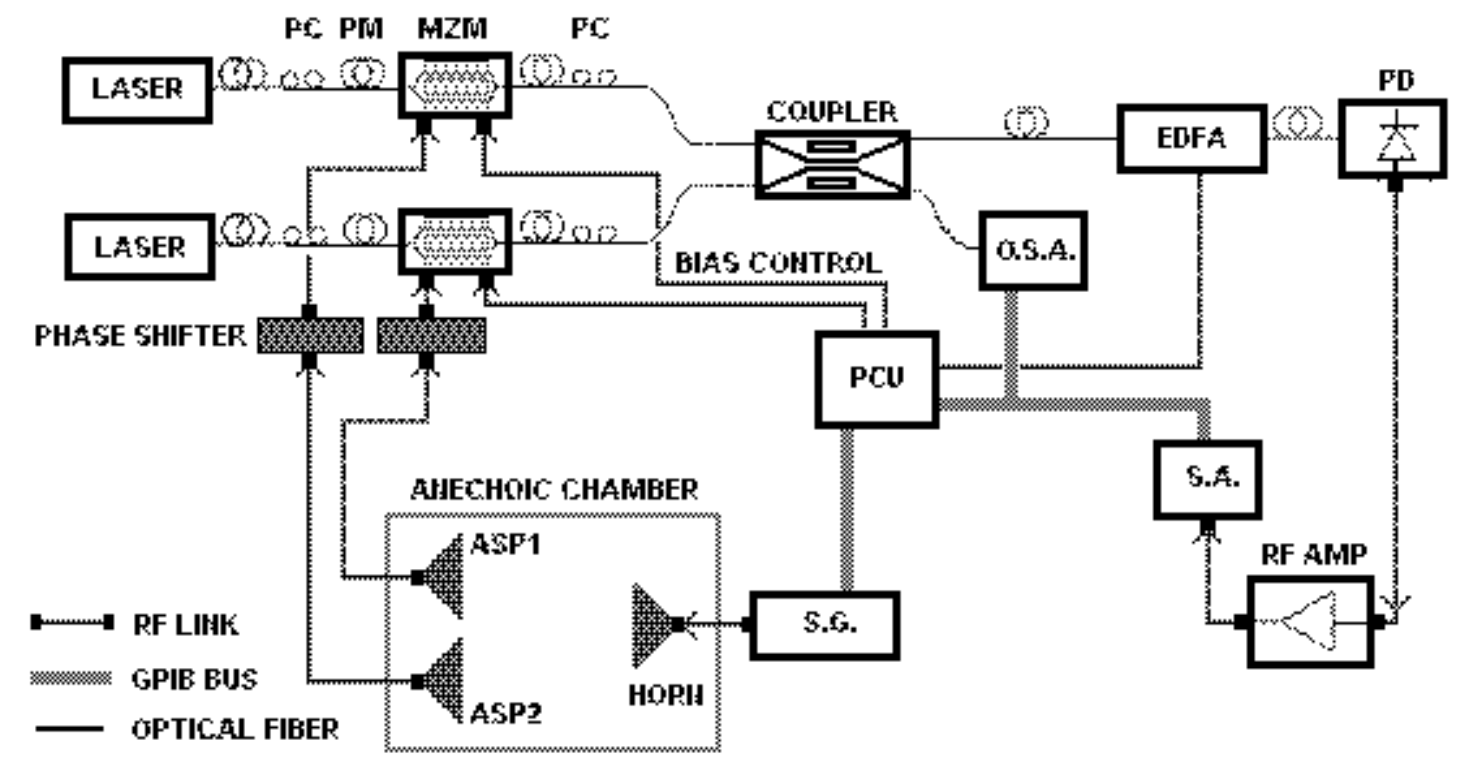

Figure 3. Microw ave Photonic Contination Setup

The gain response of each antemna was measured individually (by operating only LD1 or LD2 in Fig. 3). The grin responses are presented in Fig. 4 as MP ASP1 and MP ASP2. These can be compared with Fig 1 (RF ASP1 and RF ASP2). The essential difference is that the measurements of Fig. 1 were made with each antenna separately mounted in the centre of the chamber, while the measurements of Fig 4 were made with the antenna elements side by side. These are virtually identical indicating that the antenna interaction is minimal.

Both optical carriers (LD1 and LD2) were then connected simultaneously and the combined response from both filter/antennas was recorded. This is al so presented in Fig. 4 with different phase offsets (M1, M2, M3 and Matched). It is evident that the response at the cross-over frequency of $1.9 \mathrm{GHz}$ is sensitive to the relative phase of each arm. Empirically, the optimum phase shift (Fig. 4, Matched) was selected to provide the near ideal performance predicted in Fig 1. This response has an aver age power level of $5 \mathrm{dBi}$ with an amplitude variation of $\pm 1.5 \mathrm{~dB}$.

Fig. 5 shows the radiation patterns of the MP System at $1.5 \mathrm{GHz}, 3 \mathrm{GHz}$ and $1.9 \mathrm{GHz}$ respectively. These can be compared with the radiation patterns of Figure 2. Fig 5a) shows the radiation at $1.5 \mathrm{GHz}$ is mostly due to ASP1. When comparing Fig 5a) with Fig. 2 a), it is evident that pattern disruption over the left hemisphere from broadside. Fig. 5b) presents radiation at $3 \mathrm{GHz}$ which is primarily due to ASP2. Comparing Fig 5b) with Fig 2b), a pattern is disturbed on the right hemisphere from broadside. This is clear evidence of the influence of the two patches have on one and other when measured side by side.

Fig. 5c) presents the measured radiation pattern in the cross over region at $1.9 \mathrm{GHz}$. This pattern exhibits a narrow beam with side lobes as predicted in Fig. $2 \mathrm{c}$ ). The side lobes are approx imately $8 \mathrm{~dB}$ lower than the main lobe.

\section{DISCUSSION AHD CONCLUSIOHS}

The results show a good agreement between the RF characterization and the measured results of the MP system. We have shown that photonic combination completely isolates the two radiating elements and hence renders filter design far simpler than would be required for a diplex er [1]. Further, this configuration is scalable to even broader bandwidths with the addition of more filter/antemas.

The frequency response plots from Section II and III shows that the trends of the RF predictions and the MP measured results are very similar. The minor differences could be attributed to: the system components introduced inside the anechoic chamber; the measurement system (which does not use a synchronized reference signal); the frequency step used between measurements; the averaging function of the spectrum analyser; or the normalization factor introduced by the relative location of the reference antenna.

The radiation patterns for the individual antennas at 1.5 $\mathrm{GHz}$ and $3 \mathrm{GHz}$ and the results obtained with the MP system shows that the location of the antennas alongside each other is the only factor affecting radiation perform ance. Also, the primary cause of the distorted pattem at the cross over frequency is due to the formation of an array with the adjacent antema. This significantly modifies the radiation pattern characteristics of individual ASP antemas, as observed in the radiation pattem Figs 2 c) and 5 c). 


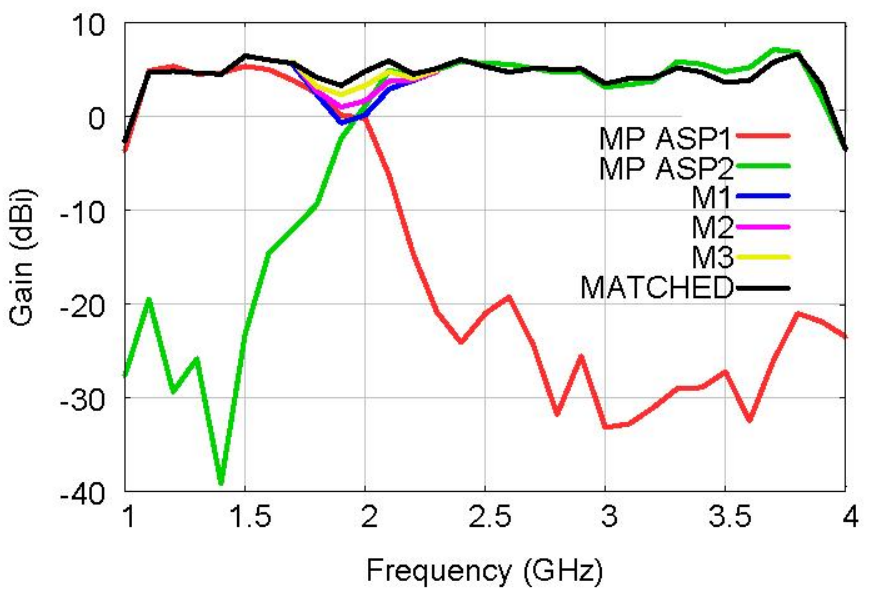

Figure 4. Microwave Photonic Combination Gain Response

\section{ACKNOWLEDGMENT}

The authors would like to thank A. Prof. James Scott for help with the filters design procedures, and David Welch, Ahmad Sami and Vikrant Kundra for their contribution to fabrication processes. We would like to acknowledge the Australian Research Council for their financial support.

\section{REFERENCES}

[1] K. Ghorbani and R. B. Waterhouse, "Ultrabroadband printed (UBP) antenna," IEEE Transactions on Antennas and Propagation, vol. 50, pp. 1697-1705, 2002.

[2] T. Kuri, K. Ikeda, , H. Toda, K. Kitayama and Y. Takahashi, "A compact remote antenna base station for microwave/millimeter-waveband radio-on-fiber systems," Lasers and Electro-Optics Society Annual Meeting (LEOS 2004), vol. 1, pp. 59-60, 2004.

[3] E. R. Lopez, L. A. Bui, K. Ghorbani, W. S. T Rowe and A. Mitchell "Ultra-broadband Photonic Patch Antenna," IEEE MTT-S International Microwave Symposium Digest, pp. 1939-1942, 2006.

[4] J. Mitola, "Software radio architecture evolution: Foundations, technology tradeoffs and architecture implications," IEICE Trans. Commun., vol. E83-B, no. 6, 2000.

[5] P. -L. Chi, S. -S. Wu and Y. -D. Lin, "Integrated antennas and diplexers for dual-band wireless local area network (WLAN) system," Asia-Pacific Microwave Conference Proceedings (APMC 2005), vol. 4, 2005.

[6] S. D. Targonski, R. B. Waterhouse and D. M. Pozar, "Design of a Wide-Band Aperture-Stacked Patch Microstrip Antenna," IEEE Transactions on Antennas and Propagation, vol. 46, pp. 1245-1251. 1998.

[7] A. Mitchell, L. A. Bui, K. Ghorbani, S. Mansoori, W. Rowe, E. Lopez and T. H. Chio, "Demonstration of a wideband photonic phased array using integrated optical RF phase shifter based on the vector sum approach," IEEE International Symposium on Phased Array Systems and Technology, pp. 199-204, 2003.

[8] H. C. Kandpal, S. Anand and J. S. Vaishya, "Experimental observation of the phenomenon of spectral switching for a class of partially coherent light," IEEE Journal of Quantum Electronics, vol. 38, pp. 336-339, 2002.
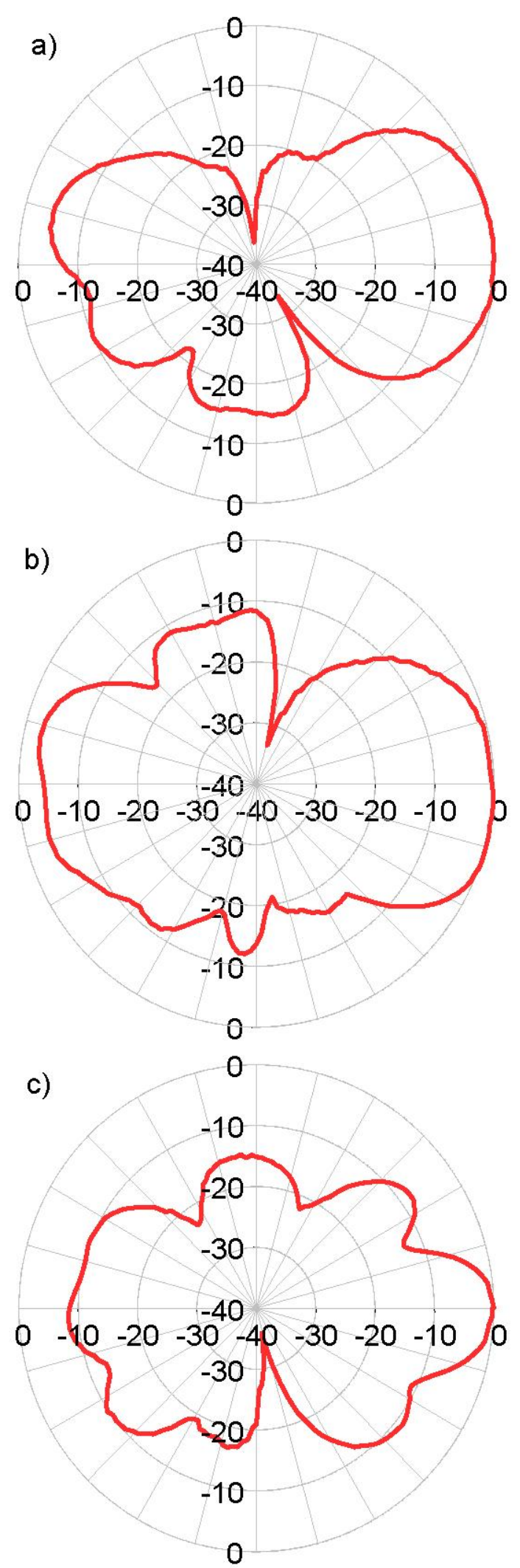

Figure 5. Measured H-plane Radiation Pattern of the MP Combination System at a) $1.5 \mathrm{GHz}$ b) $3 \mathrm{GHz}$ c) $1.9 \mathrm{GHz}$ 